\title{
ON THE DISTANCE BETWEEN ZEROES AND THE LIMIT-POINT PROBLEM
}

\author{
JURANG YAN
}

(Communicated by Kenneth R. Meyer)

\begin{abstract}
For the oscillatory equation $x^{\prime \prime}(t)+q(t) x(t)=0$, we prove some new results on the distance between consecutive zeroes and answer the questions raised by Everitt, Giertz and Weidmann [3] and by Patula [5] respectively and show that a counterexample given by Butler and Rao [1] is incorrect.
\end{abstract}

We are concerned with the differential equation

$$
x^{\prime \prime}(t)+q(t) x(t)=0
$$

where $q(t)$ is a real-valued function defined on $[a, \infty)(a \geq 0)$ and is locally integrable. A solution of (1) is said to be oscillatory if the set of its zeroes is not bounded. Since the equation is linear homogeneous, if one solution is oscillatory, all are, and hence we can say the equation is oscillatory. Equation (1) is called limit point, if at least one solution $x(t) \notin L^{2}[a, \infty)$. If any two linearly independent (and thus all) solutions are square integrable, $(1)$ is called limit circle. See [2, Ch. 9] for a description of the basic theory.

Let $q^{+}(t)=\max (q(t), 0)$. In [5] the following theorem was established.

Theorem (Patula [5]). Suppose $q^{+}(t) \in L^{p}[a, \infty), 1 \leq p<\infty$. If $(1)$ is oscillatory and if $x(t)$ is any solution, then the distance between consecutive zeros of $x(t)$ must become infinite.

As a corollary, Patula proved that if $q^{+}(t) \in L^{p}[0, \infty), 1 \leq p<\infty$, then (1) is in the limit point classification.

The purpose of this paper is to present some new results regarding this subject; in particular, we answer respectively the questions raised by Everitt, Giertz and Weidmann [3] and by Patula [5].

The following lemma $[4$, p. 345] will be needed in the proof of Theorem 1 in this paper.

Received by the editors December 2, 1988 and, in revised form, January 4, 1989.

1980 Mathematics Subject Classification (1985 Revision). Primary 34C10; Secondary 34B20.

Key words and phrases. Linear differential equation, limit point, distance between consecutive zeroes. 
Lemma 1. A necessary condition for any solution $x(t)$ of (1) to have two zeroes in $[a, b]$ is that

$$
\int_{a}^{b} q^{+}(t) d t>\frac{4}{b-a}
$$

Theorem 1. Suppose that

$$
\limsup _{t \rightarrow \infty}\left[\delta \int_{t}^{t+\delta} q^{+}(s) d s\right]<4
$$

for all $\delta>0$. If $x(t)$ is an oscillatory solution of (1), then the distance between consecutive zeroes of $x(t)$ must become infinite.

Proof. Suppose to the contrary that (1) has a solution $x(t)$ with its sequence of zeroes $\left\{t_{n}\right\}_{n=1}^{\infty}$ which has a subsequence $\left\{t_{n_{k}}\right\}_{k=1}^{\infty}$ such that $0<t_{n_{k+1}}-t_{n_{k}} \leq$ $\delta<\infty$ for some $\delta$ and all $k$. An application of Lemma 1 implies that

$$
\int_{t_{n_{k}}}^{t_{n_{k+1}}} q^{+}(s) d s>\frac{4}{t_{n_{k+1}}-t_{n_{k}}}
$$

for any $k$. Thus we have that

$$
\delta \int_{t_{n_{k}}}^{t_{n_{k+1}}} q^{+}(s) d s>4, \quad \text { for all } k .
$$

This obviously contradicts (2), and the proof of the theorem is complete.

Theorem 2. Suppose that there is a $\delta_{0}>0$ such that

$$
\lim _{t \rightarrow \infty} \int_{t}^{t+\delta_{0}} q^{+}(s) d s=0 .
$$

If $(1)$ is oscillatory and $x(t)$ is any solution, then the distance between consecutive zeroes of $x(t)$ must become infinite.

The proof of this theorem will require the following lemma.

Lemma 2. Suppose that the nonnegative function $f(t)$ is locally integrable on $[a, \infty)$. If there is a $\delta_{0}>0$ such that

$$
\lim _{t \rightarrow \infty} \int_{t}^{t+\delta_{0}} f(s) d s=0 .
$$

Then for any $\delta>0$,

$$
\lim _{t \rightarrow \infty} \int_{t}^{t+\delta} f(s) d s=0 .
$$

Proof. If $\delta \leq \delta_{0}$, proof of the lemma will be trivial. Suppose $\delta>\delta_{0}$, then there exists a positive integer $k$ such that $k \delta_{0} \geq \delta$. Thus

$$
\int_{t}^{t+\delta} f(s) d s \leq \sum_{i=0}^{k-1} \int_{t+i \delta_{0}}^{t+(i+1) \delta_{0}} f(s) d s .
$$


Let $\tau_{i}=t+i \delta_{0}, i=0,1, \ldots, k-1$, then

Consequently

$$
\lim _{t \rightarrow \infty} \int_{t+i \delta_{0}}^{t+(i+1) \delta_{0}} f(s) d s=\lim _{\tau_{i} \rightarrow \infty} \int_{\tau_{i}}^{\tau_{i}+\delta_{0}} f(s) d s=0 .
$$

$$
0 \leq \lim _{t \rightarrow \infty} \int_{t}^{t+\delta} f(s) d s \leq \sum_{i=0}^{k-1} \lim _{\tau_{i} \rightarrow \infty} \int_{\tau_{i}}^{\tau_{i}+\delta_{0}} f(s) d s=0 .
$$

The lemma is proved.

Proof of Theorem 2. From (3) and Lemma 2 we get that

$$
\lim _{t \rightarrow \infty} \delta \int_{t}^{t+\delta} q^{+}(s) d s=0
$$

for any $\delta>0$. Using Theorem 1, we know that if $x(t)$ is any solution, then the distance between consecutive zeroes of $x(t)$ must become infinite. The proof of this theorem is, therefore, complete.

Remark. Theorem 2 improves [5, Theorem 2].

Example. Let $q(t)=1 / \ln t, t>1$. For some $\delta_{0}>0$

$$
\lim _{t \rightarrow \infty} \int_{t}^{t+\delta_{0}} \frac{d s}{\ln s}=0
$$

By Theorem 2, we know that the distance between zeroes of any solution $x(t)$ tends to infinity, as $t \rightarrow \infty$. But, for any $p \geq 1, \ln t<t^{1 / 2 p}, t>1$, hence $\int_{2}^{\infty} d s / \ln ^{p} s \geq \int_{2}^{\infty} d s / \sqrt{s}=\infty$. Thus we may find that Theorem 2 in [5] is not applicable to this equation since $1 / \ln t \notin L^{p}[a, \infty), p \geq 1$.

Theorem 3. Suppose that $0<p<\infty$, and for a constant $\delta_{0}>0$

$$
\lim _{t \rightarrow \infty} \int_{t}^{t+\delta_{0}} q^{+}(s)^{p} d s=0 .
$$

If $x(t)$ is any oscillatory solution of (1), then the distance between consecutive zeroes of $x(t)$ must become infinite.

Proof. Suppose to the contrary that there exists a solution $x(t)$ of (1) with its sequence of zeroes $\left\{t_{n}\right\}_{n=1}^{\infty}$, which has a subsequence $\left\{t_{n_{k}}\right\}_{k=1}^{\infty}$ such that

$$
0<t_{n_{k+1}}-t_{n_{k}} \leq \delta, \quad k=1,2, \ldots
$$

Now, we distinguish two cases.

Case 1. $p>1$. By Lemma 1 and the Hölder inequality

$$
\begin{aligned}
4 & <\left(t_{n_{k+1}}-t_{n_{k}}\right) \int_{t_{n_{k}}}^{t_{n_{k+1}}} q^{+}(s) d s \\
& <\left(t_{n_{k+1}}-t_{n_{k}}\right)^{1+1 / i}\left(\int_{t_{n_{k}}}^{t_{n_{k+1}}} q^{+}(s)^{p} d s\right)^{1 / p} \\
& \leq \delta^{1+1 / i}\left(\int_{t_{n_{k}}}^{t_{n_{k}}+\delta} q^{+}(s)^{p} d s\right)^{1 / p}
\end{aligned}
$$


where $1 / p+1 / \gamma=1$, which contradicts (6) and Lemma 2.

Case 2. $0<p \leq 1$. Let

$$
\begin{aligned}
& E_{1}(t)=\left\{s: q^{+}(s) \leq 1, s \in\left(t, t+\delta_{0}\right)\right\}, \\
& E_{2}(t)=\left\{s: q^{+}(s)>1, s \in\left(t, t+\delta_{0}\right)\right\},
\end{aligned}
$$

and for any Lebesgue measurable set $E, m E$ is its Lebesgue measure. Thus

$$
\int_{t}^{t+\delta_{0}} q^{+}(s) d s=\int_{E_{1}} q^{+}(s) d s+\int_{E_{2}} q^{+}(s) d s .
$$

It is easy to show by (6) that $\lim _{t \rightarrow \infty} m E_{2}(t)=0$. Using this fact, we know that for any $\varepsilon>0$, there exists $T_{1}>a$ such that for all $t>T_{1} \int_{E_{2}} q^{+}(s) d s<\varepsilon / 2$. On the other hand by applying (6), we can conclude that there exists $T_{2}>a$ such that

$$
\int_{E_{1}} q^{+}(s) d s \leq \int_{t}^{t+\delta_{0}} q^{+}(s)^{p} d s<\frac{\varepsilon}{2} \quad \text { for all } t \geq T_{2} .
$$

Thus, noting (7), we obtain that

$$
0 \leq \int_{t}^{t+\delta_{0}} q^{+}(s) d s \leq \varepsilon
$$

for every $t \geq T=\max \left(T_{1}, T_{2}\right)$. Now applying Theorem 2, we see that the distance between consecutive zeroes of $x(t)$ approaches infinity as $t$ approaches infinity.

To obtain limit point result, we need the following lemma.

Lemma 3 (Patula and Wong [6]). Let (1) be the limit circle. Then (1) is oscillatory and the distance between consecutive zeroes of any solution tends to zero as $t \rightarrow \infty$

Corollary. Suppose that for $\delta_{0}>0$,

$$
\lim _{t \rightarrow \infty} \int_{t}^{t+\delta_{0}} q^{+}(s) d s=0,
$$

then (1) is in the limit point classification.

Proof. We suppose the contrary, that (1) is limit circle. Let $x(t)$ be any solution of (1). By Lemma 3, $x(t)$ is oscillatory and the distance between consecutive zeroes of $x(t)$ tends to zero, as $t \rightarrow \infty$. However, Theorem 2 means that if $x(t)$ is oscillatory, the distance between consecutive zeroes must become unbounded. This is a contradiction. Thus equation (1) must be limit point.

Remark. By Theorem 3 and Corollary, we know that

(i) Suppose $q^{+}(t) \in L^{p}[a, \infty), 0<p<1$, and if $x(t)$ is oscillatory solution, then the distance between consecutive zeroes of $x(t)$ becomes infinite;

(ii) If $q^{+}(t) \in L^{p}[a, \infty), 0<p<1$, then (1) is limit point. 
Thus we answer completely the questions raised by Everitt, Giertz and Weidmann [3] and by Patula [5] respectively. For Patula's question, a negative answer has been given in [1]. Our results imply that the counterexample of [1] is incorrect.

\section{REFERENCES}

1. G. J. Butler and V. Sree Hari Rao, Some properties of second order linear differential equations and perturbations that preserve them, SIAM J. Math. Anal. 15 (1984), 912-921.

2. E. A. Coddington and N. Levinson, Theory of ordinary differential equations, McGraw-Hill, New York, 1955.

3. W. N. Everitt, M. Giertz and J. Weidmann, Some remarks on a separation and limit-point criterion of second-order, ordinary differential expressions, Math. Ann. 200 (1973), 335-346.

4. P. Hartman, Ordinary differential equations, Wiley, New York, 1964.

5. W. T. Patula, On the distance between zeroes, Proc. Amer. Math. Soc. 52 (1975), 245-251.

6. W. T. Patula and J. S. W. Wong, An $L^{p}$-analogue of the Weyl altemative, Math. Ann. 197 (1972), 9-28.

Department of Mathematics, Shanxi University, Taiyuan, Shanxi, People's Republic OF CHINA 